\title{
Stimulation of natural killer cells with a CD137-specific antibody enhances trastuzumab efficacy in xenotransplant models of breast cancer
}

\author{
Holbrook E. Kohrt, ${ }^{1}$ Roch Houot, ${ }^{1,2,3}$ Kipp Weiskopf, ${ }^{1}$ Matthew J. Goldstein, ${ }^{1}$ Ferenc Scheeren, ${ }^{4}$ \\ Debra Czerwinski, ${ }^{1}$ A. Dimitrios Colevas, ${ }^{1}$ Wen-Kai Weng, ${ }^{1}$ Michael F. Clarke, ${ }^{4}$ \\ Robert W. Carlson, ${ }^{1}$ Frank E. Stockdale, ${ }^{1}$ Joseph A. Mollick, ${ }^{1}$ Lieping Chen, ${ }^{5}$ and Ronald Levy ${ }^{1}$ \\ 1Department of Medicine, Division of Oncology, Stanford University, Stanford, California, USA. 2Service d'Hématologie Clinique, \\ Centre Hospitalier Universitaire de Rennes, Rennes, France. ${ }^{3}$ INSERM U917, Université de Rennes 1, Rennes, France. \\ ${ }^{4}$ Institute for Stem Cell Biology and Regenerative Medicine, Stanford University, Stanford, California, USA. \\ ${ }^{5}$ Department of Immunobiology, Yale Cancer Center, New Haven, Connecticut, USA.
}

\begin{abstract}
Trastuzumab, a monoclonal antibody targeting human epidermal growth factor receptor 2 (HER2; also known as HER-2/neu), is indicated for the treatment of women with either early stage or metastatic HER2 $2^{+}$breast cancer. It kills tumor cells by several mechanisms, including antibody-dependent cellular cytotoxicity (ADCC). Strategies that enhance the activity of ADCC effectors, including NK cells, may improve the efficacy of trastuzumab. Here, we have shown that upon encountering trastuzumab-coated, HER2-overexpressing breast cancer cells, human NK cells become activated and express the costimulatory receptor CD137. CD137 activation, which was dependent on NK cell expression of the Fc $\gamma$ RIII receptor, occurred both in vitro and in the peripheral blood of women with HER2-expressing breast cancer after trastuzumab treatment. Stimulation of trastuzumab-activated human NK cells with an agonistic mAb specific for CD137 killed breast cancer cells (including an intrinsically trastuzumab-resistant cell line) more efficiently both in vitro and in vivo in xenotransplant models of human breast cancer, including one using a human primary breast tumor. The enhanced cytotoxicity was restricted to antibody-coated tumor cells. This sequential antibody strategy, combining a tumor-targeting antibody with a second antibody that activates the host innate immune system, may improve the therapeutic effects of antibodies against breast cancer and other HER2-expressing tumors.
\end{abstract}

\section{Introduction}

Of the 207,000 women diagnosed with breast cancer in the United States in 2010, one-fourth had tumors overexpressing the transmembrane receptor tyrosine kinase human epidermal growth factor receptor 2 (HER2; also known as HER-2/neu). These women comprise a disproportionate number of the 40,000 annual breast cancer deaths. Trastuzumab is a humanized $\mathrm{mAb}$ targeting HER2. Despite improving the outcome for this poorprognostic group of patients, response rates in metastatic breast cancer to trastuzumab as monotherapy are limited, consisting of approximately $10 \%-15 \%(1)$.

Multiple strategies have been investigated to enhance the antitumor activity of trastuzumab, which is due, at least in part, to antibody-dependent cellular cytotoxicity (ADCC) (2-5). ADCC is dependent upon immune effector cells, mainly NK cells, binding via their $\mathrm{Fc}$ receptor (FcrRIII, CD16) to the IgG1 Fc, heavy-chain portion of trastuzumab (3). This leads to the activation of the NK cells, release of their cytotoxic granules, and lysis of the trastuzumab-bound breast cancer cell (6). Clinical results have shown that patients harboring an FcyRIIIA polymorphism with higher NK affinity for IgG1 have a better response to trastuzumab, further

Authorship note: Holbrook E. Kohrt and Roch Houot contributed equally to this work.

Conflict of interest: The authors have declared that no conflict of interest exists. Citation for this article: J Clin Invest. 2012;122(3):1066-1075. doi:10.1172/JCI61226 supporting the hypothesis that ADCC, including its mediators, is an important in vivo mechanism of trastuzumab action $(7,8)$. Additional supporting clinical data demonstrated that responders to neoadjuvant trastuzumab exhibited a 4-fold increase in antibody-dependent lytic activity from isolated PBMCs compared with that of nonresponders (4). Therefore, augmenting ADCC could increase the clinical efficacy of trastuzumab therapy.

Selectively targeting activated NK cells at the tumor site would be an attractive strategy to improve ADCC without incurring the systemic toxicity of global NK cell stimulation, such as that observed with systemic IL-2 or IL-12 $(9,10)$. Recently, it was shown that human NK cells upon Fc-receptor triggering, such as the interaction with antibody-bound tumor cells, upregulate the inducible costimulatory molecule CD137 (11). Once induced to express CD137, we hypothesize that the killing function of these activated NK cells can be enhanced by their exposure to an agonistic mAb against CD137, leading to improved antitumor activity. In the current study, we investigate the hypothesis that an agonistic $\mathrm{mAb}$ against CD137 can enhance the killing of human breast cancer cells by trastuzumab both in vitro and in vivo.

\section{Results}

Human HER2-expressing tumor cells coated with trastuzumab induce the expression of CD137 on buman NK cells. Purified NK cells from healthy human subjects were incubated with trastuzumab and breast cancer cell lines (BT474M1, HER18, or SKBR3) expressing 
A
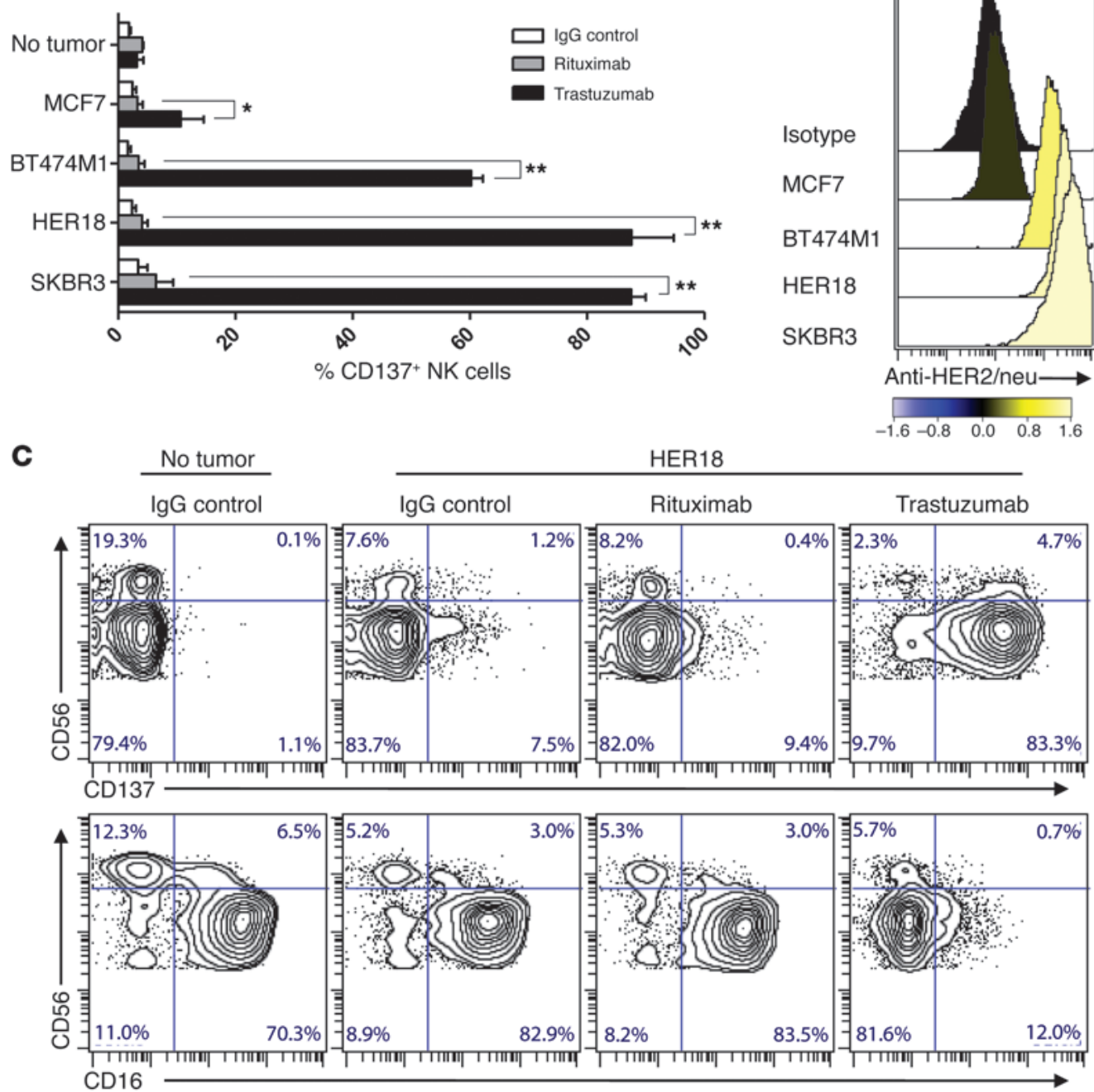

B

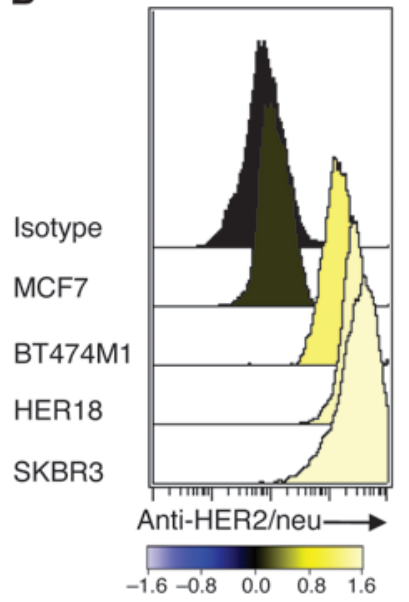

$3 \%$ $7 \%$

\section{Figure 1}

Trastuzumab induces CD137 upregulation on human NK cells following exposure to HER2-overexpressing tumor cells. Purified NK cells from the peripheral blood of 3 healthy donors were analyzed for CD137 expression after 24-hour culture with breast cancer cell lines or no tumor and IgG control, rituximab, or trastuzumab. (A) Percentage of CD137+ cells among CD3-CD56 ${ }^{+} \mathrm{NK}$ cells from 3 healthy donors cultured with MCF7, BT474M1, HER18, and SKBR3 breast cancer cell lines. ${ }^{\star} P=0.046$; ${ }^{* *} P<0.001$. Data are shown as mean \pm SEM. (B) HER2 surface expression on breast cancer cell lines, with histograms colored according to the $\log _{10}$-fold increase in MFI of breast cancer cell line relative to isotype. (C) CD137 and CD16 expression on NK cell subsets CD3-CD56 ${ }^{\text {bight }}$ and CD3-CD56 dim from a representative healthy donor after 24-hour culture with IgG control alone, HER18 and IgG control, HER18 and rituximab, or HER18 and trastuzumab. Percentages of total NK cells per quadrant are indicated.
HER2. This resulted in robust upregulation of CD137 expression. In contrast, incubation of the same human NK cells in the absence of tumor cells or in the presence of HER2-expressing tumor cells and a nonbinding $\mathrm{mAb}$ (rituximab) had little effect on CD137 expression (Figure 1A). No induction of CD137 occurred on NK cells following incubation of breast cancer cell lines with trastuzumab in the presence of a breast cancer cell line that does not overexpress HER2 (MCF7) (Figure 1, A and B). Similarly, trastuzumab D265A, a trastuzumab variant that does not bind human FcyRs, abrogated the increase in CD137 expression on NK cells following exposure to trastuzumab-coated HER2-expressing tumor cells (Supplemental Figure 1; supplemental material available online with this article; doi:10.1172/ JCI61226DS1). CD137 upregulation occurred preferentially among CD56 $6^{\mathrm{dim}}$ in comparison with CD56 ${ }^{\mathrm{hi}} \mathrm{NK}$ cells (Figure $1 \mathrm{C}$ ). The induction of CD137 peaked after 24 hours and was associated with a concurrent decrease in the expression of Fc $\gamma$ RIII (CD16) (Figure 1C). Despite a similar decrease in NK cell expression of CD16 following culture with trastuzumab-bound HER2-expressing tumor cells (Supplemental Figure 2A), NK cells from healthy donors with high-affinity polymorphisms of Fc $\gamma$ RIIIA-158
(V/V or F/V) expressed increased levels of CD137 compared with NK cells from donors with a low affinity FcrRIIIA-158 (F/F) polymorphism (Supplemental Figure 2B).

An agonistic anti-CD137 mAb increases trastuzumab-mediated NK cell cytokine secretion and trastuzumab-dependent NK cell-mediated cytotoxicity. NK cells from healthy donors were cocultured with trastuzumab-bound HER2-expressing breast cancer cells for 24 hours. Subsequently, the activated NK cells were reisolated and combined with breast cancer cells under different antibody conditions. The function of these activated NK cells was investigated by measuring secretion of IFN- $\gamma$ and lysis of breast cancer cells. Anti-CD137 agonistic mAb significantly increased trastuzumab-induced IFN- $\gamma$ secretion when incubated with 2 of 3 HER2-overexpressing breast cancer cell lines (SKBR3 and HER18) (Figure 2, A-D). Moreover, the anti-CD137 mAb enhanced the ability of these activated NK cells to kill trastuzumab-coated tumor cells (Figure 2, E-H). AntiCD137 mAb alone had minimal effect in the absence of both trastuzumab and HER2-overexpressing breast cancer cells. To confirm the enhanced cytotoxicity, ${ }^{51} \mathrm{Cr}$-labeled breast cancer cell lines were cultured with NK cells, both unpurified (Supplemental Figure 3) and purified (Figure 3, A-D), following activation in the 
A

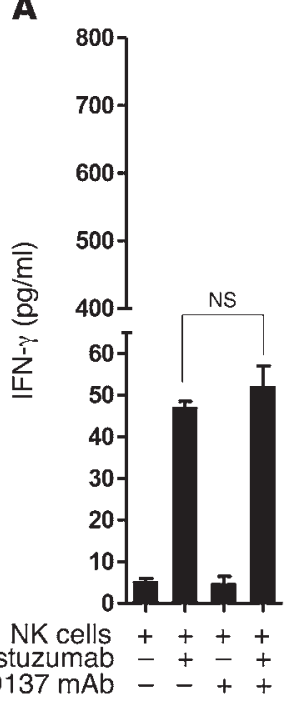

E

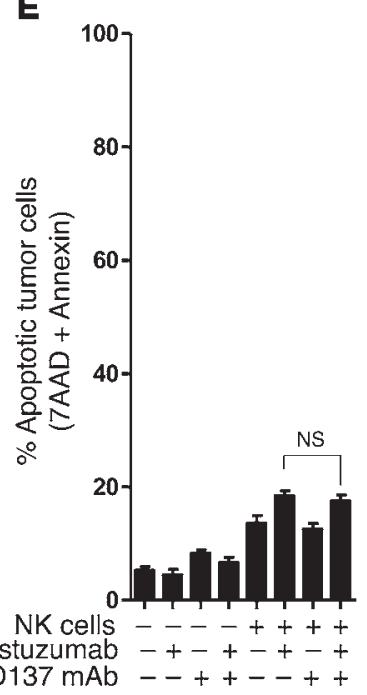

B

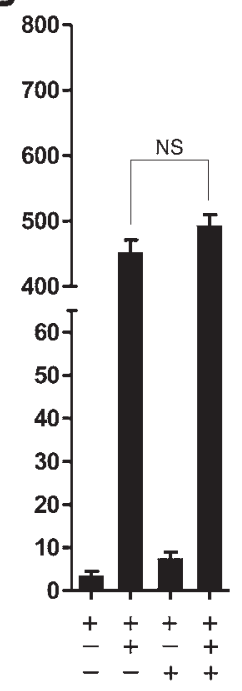

F

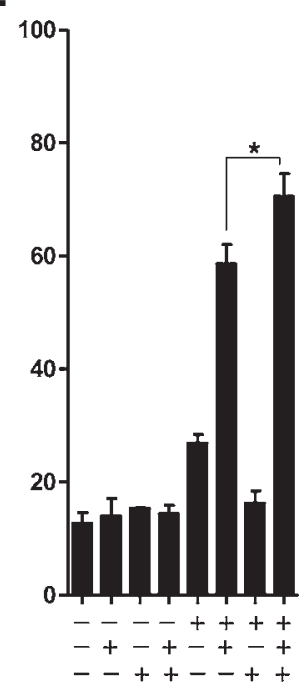

C

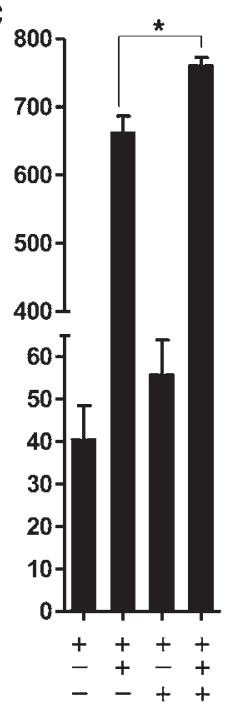

G

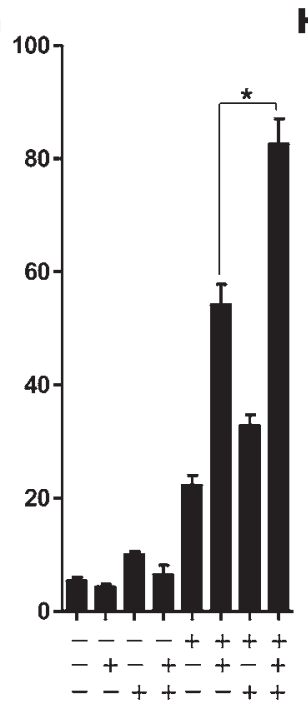

D

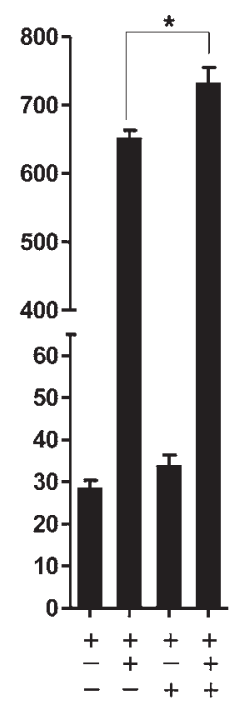

H

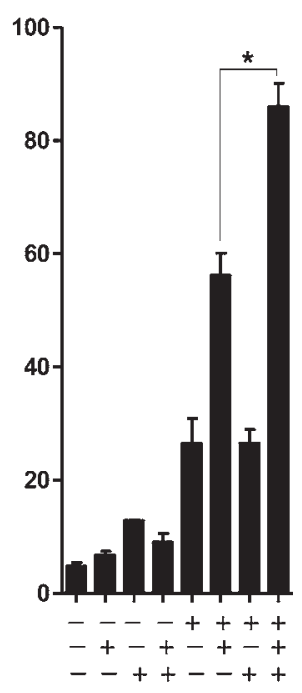

Figure 2

Anti-CD137 agonistic mAbs increase trastuzumab-mediated NK cell cytokine secretion and cytotoxicity on tumor cells as assayed by cell viability. To evaluate NK cell function, purified NK cells were isolated from 3 independent, healthy donor PBMCs and cultured for 24 hours together with trastuzumab $(10 \mu \mathrm{g} / \mathrm{ml})$ and irradiated (50 Gy) breast cancer cells (SKBR3) at a ratio of 1:1. After 24 hours, NK cells were isolated by negative selection and assessed for purity (>90\% purity as defined by CD3-CD56+ flow cytometry) and activation (>50\% expression of CD137). Breast cancer cell lines including MCF7 (A and E), BT474M1 (B and F), HER18 (C and G), and SKBR3 (D and H) were cultured for 18 hours with preactivated, purified NK cells in medium alone, or with anti-CD137 mAb (BMS-663513, $10 \mu \mathrm{g} / \mathrm{ml})$ alone, trastuzumab (10 $\mu \mathrm{g} / \mathrm{ml})$ alone, or trastuzumab plus anti-CD137 mAbs (both at $10 \mu \mathrm{g} / \mathrm{ml}$ ), and supernatant was harvested and analyzed by ELISA for IFN- $\gamma(\mathbf{A}, \mathrm{MCF} 7, P=0.39$; B, BT474M1, $P=0.16$; C, HER18, ${ }^{*} P=0.017$; D, SKBR3, $\left.{ }^{*} P=0.034\right)$. Cells were washed and incubated with annexin $V$ and $7-A A D$ to determine percentage of apoptotic tumor cells by annexin V and 7-AAD staining (E, MCF7, $P=0.43 ; \mathbf{F}, \mathrm{BT} 474 \mathrm{M} 1,{ }^{*} P=0.031 ; \mathrm{G}, \mathrm{HER} 18,{ }^{*} P<0.001 ; \mathrm{H}, \mathrm{SKBR} 3$, $\left.{ }^{*} P<0.001\right)$. Data are shown as mean \pm SEM.

presence or absence of trastuzumab and anti-CD137 mAb. Again, the combination of anti-CD137 antibodies with trastuzumab induced enhanced ADCC relative to treatment with trastuzumab alone using both unpurified and purified NK cells. This effect was observed only against HER2-overexpressing breast cancer cell lines, confirming that the killing was due to ADCC, even though the NKs had been preactivated.

To investigate whether augmented antibody-mediated cytotoxicity of activated NK cells is restricted to the antibodycoated tumor cell used for NK cell activation, cytotoxicity against
HER2-expressing breast cancer and CD20+ lymphoma cells was compared using NK cells activated by trastuzumab-coated breast cancer or rituximab-coated lymphoma cells. Activated NK cells lysed antibody-coated tumor cells with augmented cytotoxicity independently of antibody-coated tumor cells used for activation. Anti-CD137 mAbs significantly increased trastuzumab-mediated breast cancer cytotoxicity of NK cells activated by culture with either trastuzumab-coated breast cancer or rituximab-coated lymphoma cells (Supplemental Figure 4, A-D). Similar enhancement of rituximab-mediated lymphoma cytotoxicity was observed using 
A

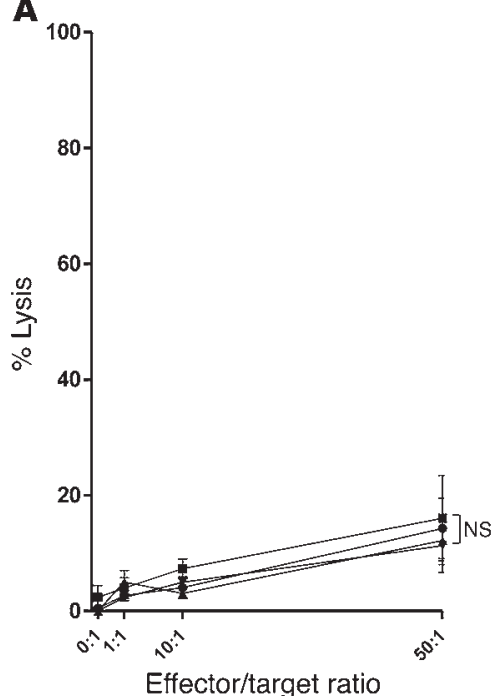

C

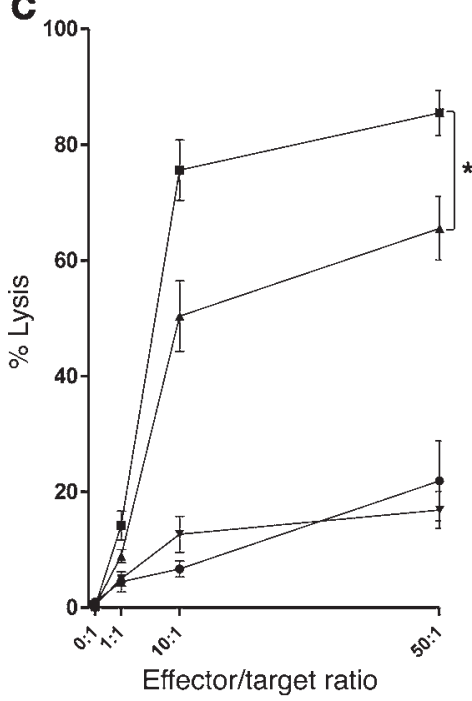

B

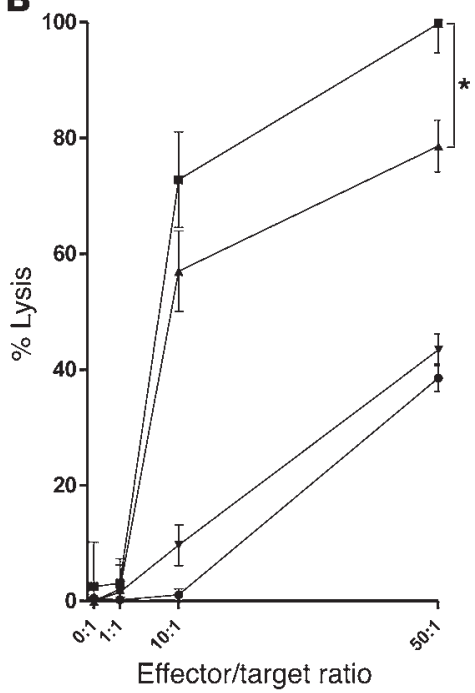

D

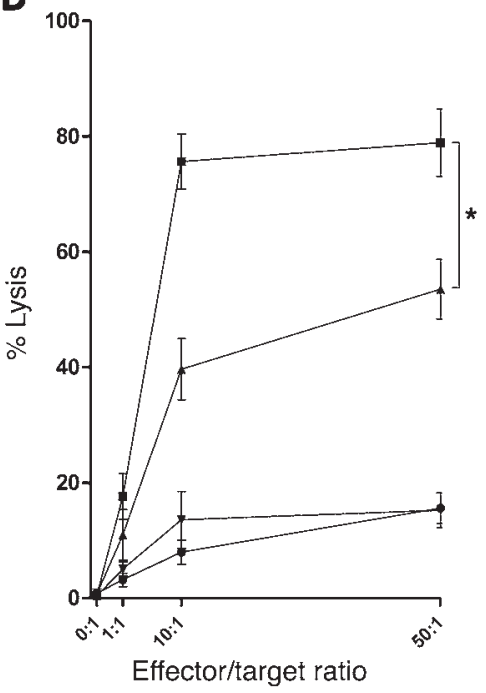

Figure 3

Anti-CD137 agonistic mAb increases trastuzumab-mediated NK cell cytotoxicity on tumor cells as assayed by chromium release. To evaluate NK cell cytolytic function, healthy PBMCs were cultured for 24 hours together with trastuzumab $(10 \mu \mathrm{g} / \mathrm{ml})$ and irradiated (50 Gy) breast cancer cells (SKBR3) at a ratio of 1:1. After 24 hours, NK cells were isolated by negative selection and assessed for purity (>90\% purity as defined by $\mathrm{CD}^{-} \mathrm{CD} 56^{+}$flow cytometry) and activation ( $>50 \%$ expression of CD137). Chromium-labeled breast cancer cell lines including MCF7 (A), BT474M1 (B), HER18 (C), and SKBR3 (D) were cultured for 4 hours with preactivated, purified NK cells in medium alone or with anti-CD137 mAbs (BMS-663513, $10 \mu \mathrm{g} / \mathrm{ml})$ alone, trastuzumab $(10 \mu \mathrm{g} / \mathrm{ml})$ alone, or trastuzumab plus anti-CD137 mAbs. Shown are percentages of lysis of target cells by chromium release at varying effector (activated NK cells)/target cell ratios cultured with medium alone (circles), anti-CD137 (inverted triangles), trastuzum$\mathrm{ab}$ (triangles), or trastuzumab and anti-CD137 (squares) antibodies $\left(\mathbf{A}, P=0.67 ; \mathbf{B},{ }^{*} P=0.006\right.$; $\mathbf{C},{ }^{*} P=0.041$; D, $\left.{ }^{\star} P=0.031\right)$. Data are shown as mean \pm SEM.
NK cells activated by either trastuzumab-coated breast cancer or rituximab-coated lymphoma cells. Finally, to determine whether anti-CD137 mAbs enhance cytotoxicity in the setting of trastuzumab resistance, cytotoxicity against a breast cancer cell line intrinsically resistant to trastuzumab (with maintained expression of HER2, HCC1569) was performed with maintained efficacy observed (Supplemental Figure 5).

Anti-CD137 agonistic mAbs enhance anti-breast cancer activity of trastuzumab in vivo. The in vivo ability of anti-CD137 mAbs to enhance trastuzumab's activity was tested in a xenotransplanted human breast cancer (BT474M1 cell line) model in athymic mice. These mice have fully competent NK cells, while lacking functional $\mathrm{T}$ cells. Anti-CD137 mAbs alone had no effect on tumor growth, while trastuzumab had a modest effect (Figure 4, A and C). We simultaneously explored the effect of trastuzumab and antiCD137 mAbs in various schedules. Since maximal upregulation of CD137 required 24 hours of NK cell exposure to trastuzumab-coated cells in vitro, we aimed to determine the importance of injection sequence. Trastuzumab was injected on day 3 (after tumor inoculation) and anti-CD137 mAb was injected on either day 2,3 , or 4 . Treatment was repeated in all groups 14 days fol- lowing the first injection. Of the 3 combination sequences tested, trastuzumab followed by anti-CD137 mAbs resulted in the greatest reduction in tumor size and mortality with the opposite order, anti-CD137 mAbs followed by trastuzumab, having the least effect on both tumor growth (Figure 4A) and survival (Figure 4B).

Since trastuzumab followed by anti-CD137 antibodies demonstrated more potent antitumor activity in vivo, we chose this injection schedule for subsequent experiments. When the treatment regimen was repeated weekly for 3 weeks, the combination treatment resulted in prolonged control of tumor growth and a significant improvement in survival (Figure 4, C and D).

Enhancement of the therapeutic activity of trastuzumab by anti-CD137 $m A b$ is specific to HER2-expressing tumors. To determine whether the synergy between anti-HER2 and anti-CD137 mAbs requires specific recognition by the antitumor antibody, 2 tumors differing in HER2 expression were inoculated s.c. in individual mice. On the left flank, mice were inoculated with MCF7 cells, which are HER2 low to negative. On the right flank, mice were inoculated with HER18 cells, a transduced derivative of MCF7 cells that stably overexpress HER2 (Figure 5A and ref. 12). Mice then received either trastuzumab monotherapy on day 3 or the combination 
A
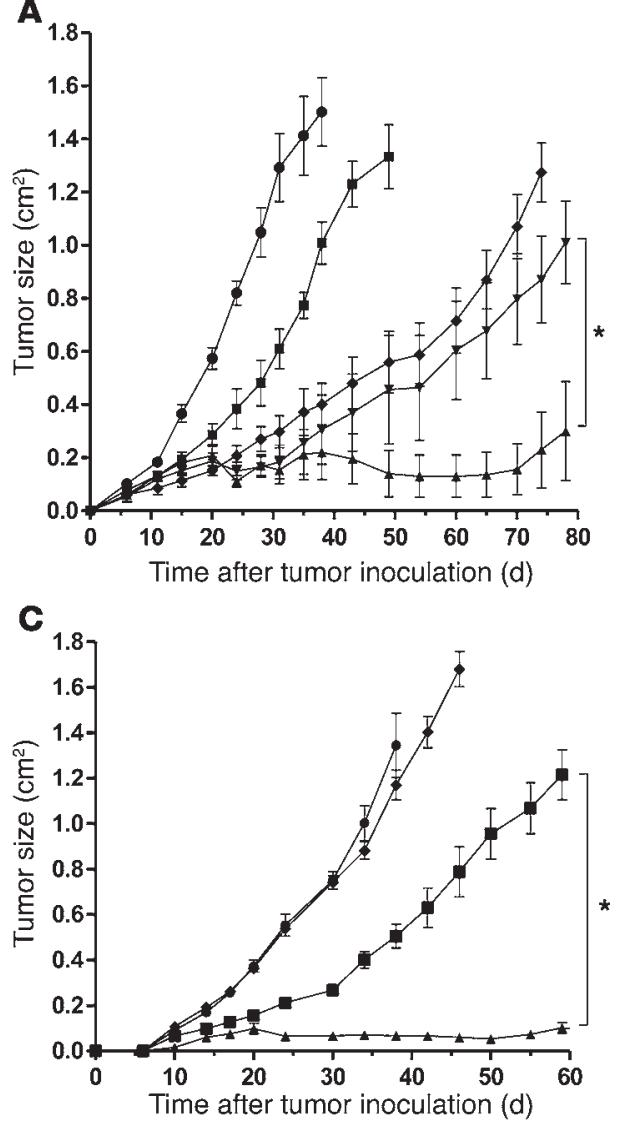

B

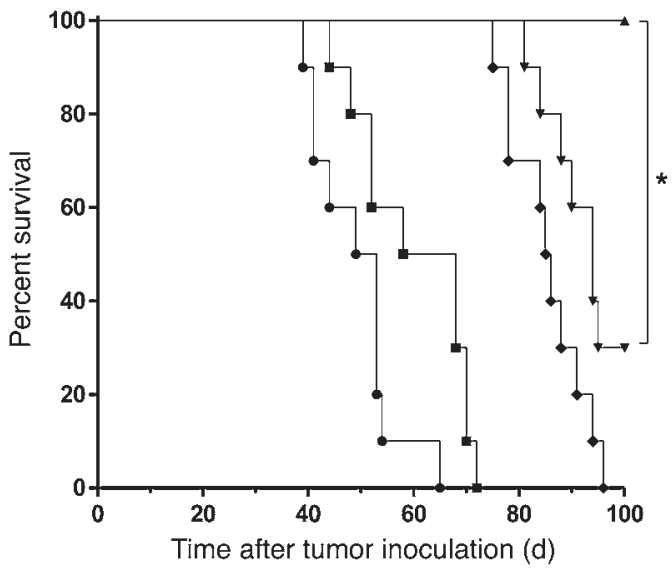

D

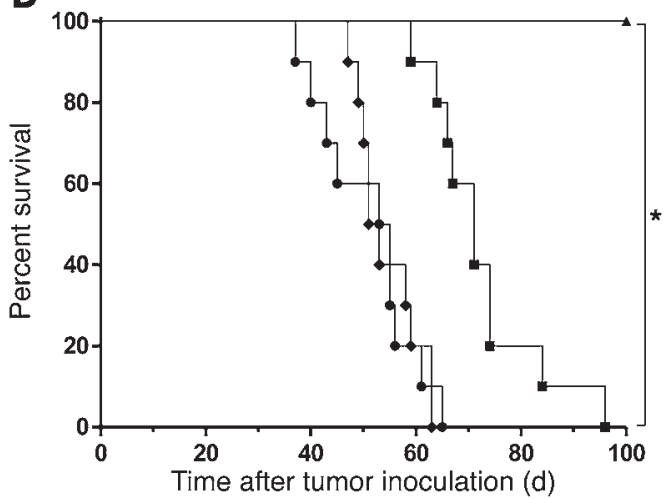

Figure 4

Anti-CD137 agonistic mAbs enhance anti-breast cancer activity of trastuzumab in vivo. nu/nu mice were inoculated with $5 \times 10^{6}$ BT474M1 breast tumor cells, s.c., on the abdomen 1 day after s.c. injection of $0.72 \mathrm{mg} / 60 \mathrm{~d}$ release $\beta$-estradiol pellet (A-D). (A and $\mathbf{B})$ After tumor inoculation, mice received 2 cycles of treatment separated by 14 days with either rat IgG control on days 3 and 17 (circles), trastuzumab antibody on days 3 and 17 (squares), trastuzumab antibody on days 3 and 17 and anti-CD137 antibody on days 2 and 16 (diamonds), trastuzumab antibody on days 3 and 17 and anti-CD137 antibody on days 3 and 17 (inverted triangles), or trastuzumab antibody on days 3 and 17 and anti-CD137 antibody on days 4 and 18 (triangles). Mice (10 per group) were then monitored for tumor growth $\left(\mathbf{A},{ }^{*} P<0.001\right)$ and overall survival $\left(\mathbf{B},{ }^{*} P=0.001\right)$. (C and D) To determine efficacy of increased frequency of treatment using the superior combination regimen, mice received 3 weekly treatments with either rat IgG control starting on day 3 (circles), trastuzumab antibody starting on day 3 (squares), anti-CD137 antibody starting on day 4 (diamonds), or trastuzumab antibody starting on day 3 and anti-CD137 antibody starting on day 4 (triangles) with treatment repeated weekly for a total of 3 weeks. Mice (10 per group) were then monitored for tumor growth $\left(\mathbf{C},{ }^{*} P<0.001\right)$ and overall survival (D, $\left.{ }^{*} P=0.003\right)$. Data are shown as mean \pm SEM.

of trastuzumab on day 3 and anti-CD137 mAb on day 4. This sequential therapy was repeated weekly for 3 successive weeks. As expected, only the HER2-overexpressing tumors responded to monotherapy with trastuzumab. The addition of anti-CD137 $\mathrm{mAbs}$ resulted in an enhanced therapeutic effect, but only against the HER2-positive tumor that could be targeted by the anti-HER2 mAbs (Figure 5, B and C).

Anti-CD137 mAbs enhance the therapeutic activity of trastuzumab against a buman HER2-overexpressing primary breast tumor. Since the combination of trastuzumab and anti-CD137 antibodies demonstrated increased efficacy against HER2-overexpressing cell lines, we next aimed to assess efficacy of the combination therapy using a primary patient xenotransplant model. A primary HER2-overexpressing breast tumor was successfully engrafted into sublethally irradi- ated SCID mice. After tumors were established, the mice were randomized into treatment groups that received one of the following: rat IgG control, trastuzumab, anti-CD137 $\mathrm{mAb}$, or trastuzumab followed 24 hours later by anti-CD137 $\mathrm{mAb}$ and repeated weekly for a total of 3 weeks. The combination of trastuzumab and antiCD137 mAbs was superior to trastuzumab alone, significantly reducing tumor growth and prolonging survival (Figure $5, \mathrm{D}$ and $\mathrm{E}$ ).

Increased expression of CD137 on circulating $N K$ cells in patients receiving trastuzumab therapy. We identified 11 patients with HER2-expressing breast cancer receiving trastuzumab therapy. PBMCs were isolated prior to and following treatment. Samples were collected at 4 hours, 24 hours, and 7 days following completion of trastuzumab infusion (Figure 6A). Reduced expression of CD16 hi and increased CD137 expression at short interval time points (24 hours) coincided with a transient loss of circulating NK cells. Seven days following trastuzumab infusion, the percentage of PBMCs that were circulating NK cells as well as expression of CD16 and $\mathrm{CD} 137$ were similar to baseline. Across the 11-patient cohort, the mean NK cell CD137 expression level at baseline, $4.4 \%$, was similar at 4 hours after trastuzumab infusion, $4.9 \%$, with a gradual, but significant, increase in CD137 expression at 24 hours, 9.6\% (Figure 6B). Grouping all post-trastuzumab infusion samples in comparison with baseline, NK cell CD137 expression increased approximately 2-fold. Interestingly, the greatest magnitude of increase in CD137 expression occurred in 2 patients receiving their first doses of trastuzumab.

\section{Discussion}

One in 5 women with HER2-overexpressing breast cancer will relapse despite trastuzumab therapy (13). To address this, multiple strategies have been investigated to enhance trastuzumab's activity and circumvent resistance $(14,15)$. Strategies currently in various stages of clinical testing have included a small molecule tyrosine kinase inhibitor, generation of bispecific antibodies, antibodytoxin conjugates, dimerization and sheddase inhibitors, and HER2 
A

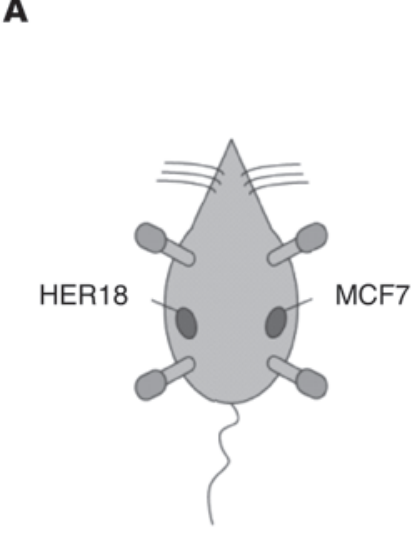

C

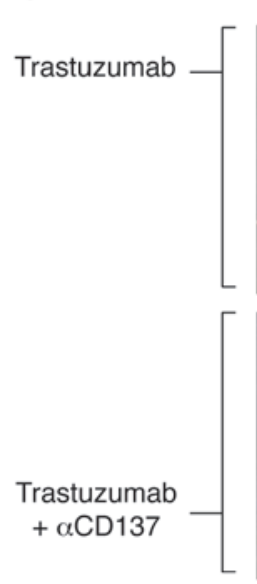

D

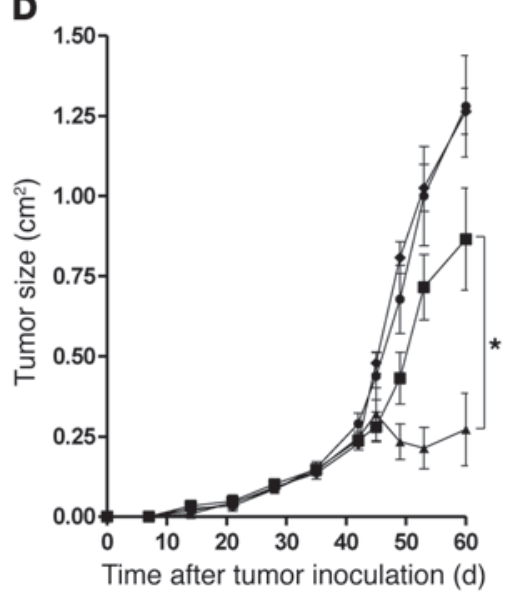

Day 25
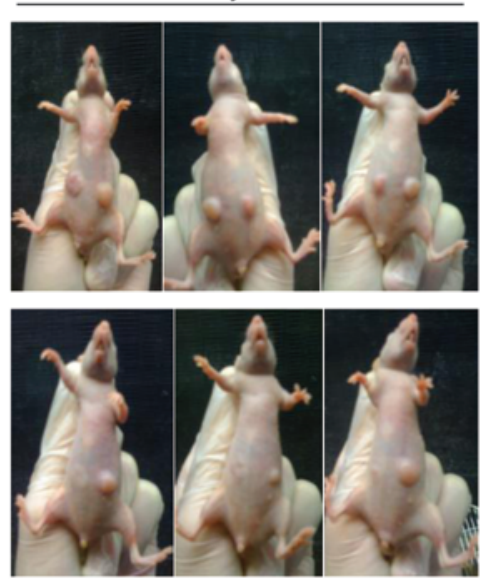

E
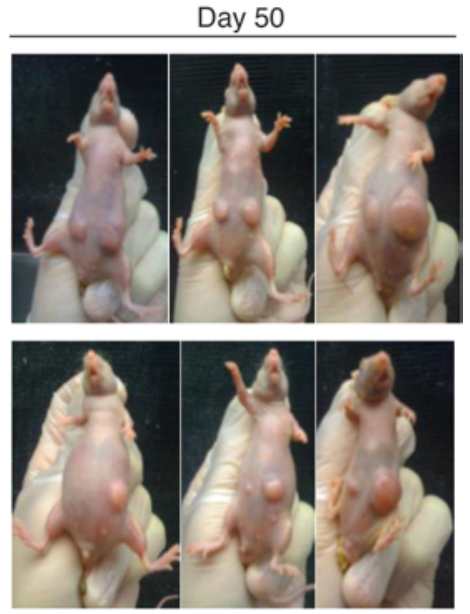

B

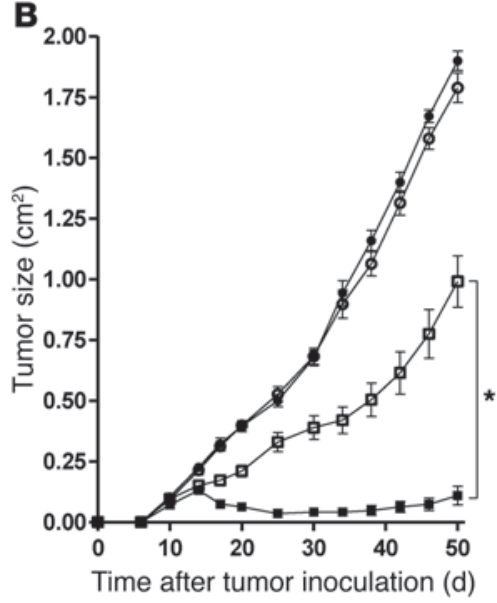

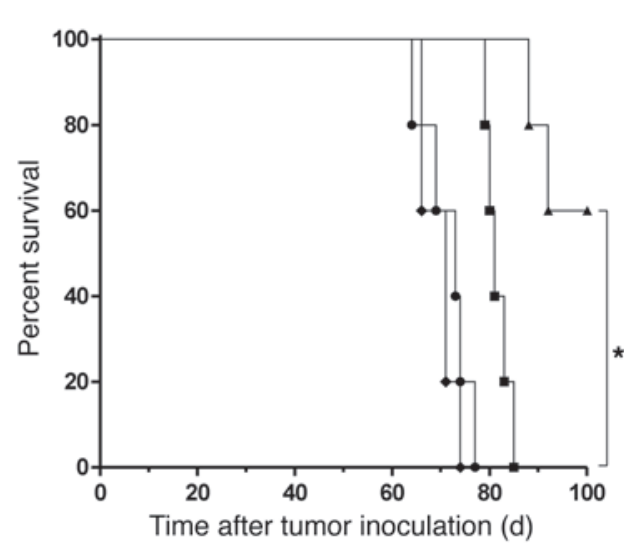

Figure 5

Anti-CD137 agonistic mAb enhances anti-breast cancer activity of trastuzumab in vivo while retaining HER2 specificity against HER2-overexpressing breast cancer cell lines and a primary breast tumor. nu/nu mice were inoculated with $5 \times 10^{6}$ MCF7 breast tumor cells S.c. on the left flank and $5 \times 10^{6}$ HER18 breast tumor cells s.c. on the right flank $(\mathbf{A})$. (A-C) After tumor inoculation, mice received either trastuzumab on day 3 or trastuzumab on day 3 and anti-CD137 antibody on day 4, with each treatment repeated weekly for a total of 3 weeks. (B) Mice (10 per group) were monitored for tumor growth of MCF7 on the left flank (white circles) and HER18 on the right flank (white squares) when treated with trastuzumab, and MCF7 on the left flank (black circles) and HER18 on the right flank (black squares) when treated with trastuzumab and antiCD137 mAbs ( $\left.{ }^{*} P<0.001\right)$. (C) Representative mice (3 of 10 per group) at 25 and 50 days after tumor inoculation. SCID mice were inoculated with $1 \times 10^{6} \mathrm{HER}^{+}$primary breast tumor cells (SU-258) by intramammary injection 24 hours after 200 cGy total body irradiation (TBI). (D and E). On day 40, mice were randomized to 1 of 4 groups (5 mice per group) including IgG control with treatment on day 40 (circles), trastuzumab on day 40 (squares), anti-CD137 mAbs on day 41 (diamonds), or trastuzumab on day 40 and anti-CD137 mAbs on day 41 (triangles). Treatment was repeated weekly in each group for a total of 3 treatments. Mice were monitored for tumor growth $\left(\mathbf{D},{ }^{*} P=0.016\right)$ and overall survival $(\mathbf{E}$, $\left.{ }^{\star} P=0.002\right)$. Data are shown as mean \pm SEM.

vaccines (16-20). Here, we present an alternative approach aiming at enhancing trastuzumab-mediated ADCC by stimulation of NK cells with an anti-CD137 agonistic mAb. Our results support the following therapeutic strategy. First, trastuzumab localizes to the site of the tumor and activates surrounding NK cells via Fc-Fc $\gamma \mathrm{R}$ interaction, which increases NK cell expression of CD137. Subsequent administration of an anti-CD137 agonistic mAb stimulates the activated NK cells to enhance their cytotoxic function while maintaining specificity to lysis of trastuzumab-bound, HER2overexpressing tumor cells.

CD137 is an inducible, costimulatory molecule expressed on activated CD4 and CD8 T cells, with the majority of prior work focusing on the use of CD137 mAbs or ligands to increase the proliferation and survival of T cells (21). Impressive preclinical activity has been documented with anti-CD137 antibodies in solid tumor models, including breast cancer, sarcoma, glioma, colon carcinoma, myeloma, and mastocytoma. These results have motivated clinical trials of agonistic anti-CD137 mAb monotherapy (21-23). CD137 expression, however, is also observed on NK cells, monocytes, dendritic cells, and nonhematopoietic cells, where it has only recently been demonstrated to have functional significance $(24,25)$. We have previously shown that the antilymphoma activity of anti-CD137 agonistic mAb monotherapy requires both CD8 T cells and NK cells (26). Although in the setting of acute myeloid leukemia, expression of CD137 ligand by the leukemic blasts has been shown to impair NK cell spontaneous cytotoxicity (27), additional mouse and human studies have observed enhanced NK cell function, including survival and spontaneous cytotoxicity against both hematopoietic and solid 
A

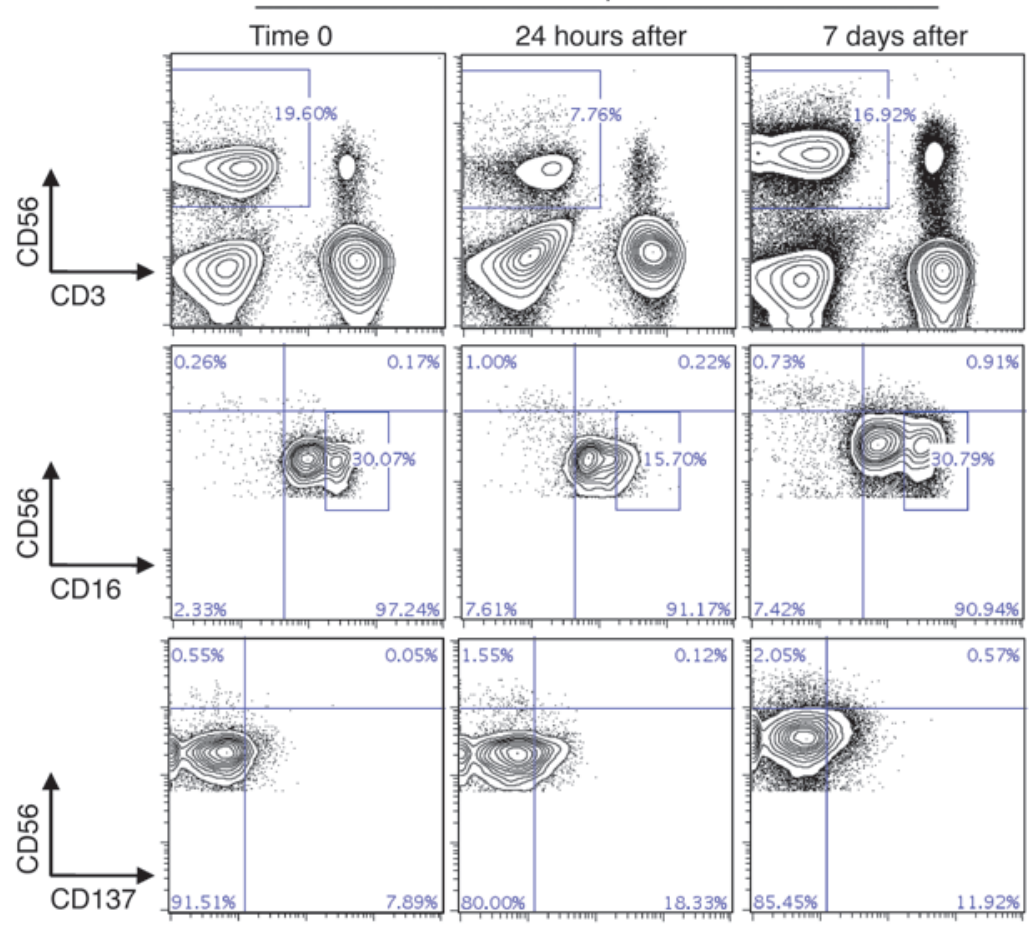

B

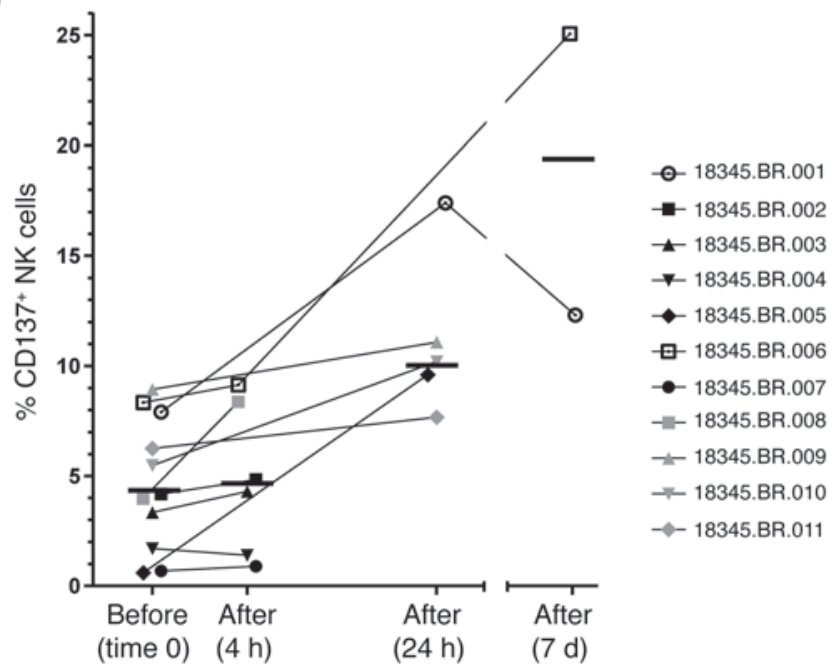

Timepoint before/after trastuzumab infusion

\section{Figure 6}

Increased expression of CD137 on circulating NK cells in patients receiving trastuzumab therapy. PBMCs were isolated from 11 patients with HER2-expressing breast cancer prior to and following trastuzumab treatment. (A) CD56 and CD3 expression on circulating lymphocytes and CD16 and CD137 expression on NK cell subsets CD3-CD56 ${ }^{\text {bight }}$ and CD $3^{-}$CD56 $6^{\text {dim }}$ from a single representative patient at 3 time points, including prior to trastuzumab, and 24 hours and 7 days following trastuzumab. (B) Percentage of CD137+ cells among circulating CD3-CD56+ NK cells from 11 patients prior to and following trastuzumab, including 2 patients receiving their first dose of trastuzumab (patients 18345.BR.001 [white circles] and 18345.BR.006 [white squares]). Data are shown as mean \pm SEM.

tumors (28-31). We recently reported that rituximab-induced ADCC of CD20-expressing lymphoma could be augmented by anti-CD137 agonistic mAbs in multiple mouse models (32).
We show that exposure of human NK cells to trastuzumab-bound, HER2-overexpressing breast cancer results in marked CD137 expression, dependent upon and influenced by the affinity of $\mathrm{Fc}-\mathrm{Fc} \gamma \mathrm{R}$ binding. Our findings support prior studies using immobilized IgG1- or rituximab-coated lymphoma cells to induce CD137 expression $(11,32)$. Notably, CD137 induction occurs predominantly among the CD56 ${ }^{\mathrm{dim}}$ subset of NK cells known to mediate ADCC, compared with CD56 $6^{\text {hi }} \mathrm{NK}$ cells, which are responsible for secretion of cytokines such as IFN- $\gamma$ (33). We observed a concurrent downregulation of CD16 among the CD56 ${ }^{\mathrm{dim}}$ subset, supporting the internalization of the Fc $\gamma \mathrm{R}$ following Fc-Fc $\gamma \mathrm{R}$ binding $(34,35)$. Fc $\gamma$ RIII polymorphisms predicted amplitude of CD137 upregulation, with increased CD137 expression following Fc-Fc $\gamma \mathrm{R}$ binding of high-affinity polymorphisms. In vivo, we expect NK cells in contact with trastuzumab-bound tumor cells to be activated regardless of anatomic site, such as within the primary tumor, tumor-involved lymph nodes, or at distant sites of metastatic breast cancer. In a small series of peripheral blood patient samples obtained prior to and following trastuzumab, downregulation of CD16 and increased expression of CD137 occurred approximately 4 to 24 hours after trastuzumab infusion, with marked heterogeneity in magnitude of change. Timing after trastuzumab infusion, prior exposure to trastuzumab, burden of disease, and genetic polymorphism of FcyRIIIa may contribute to the heterogeneity. As efficacy of antiCD137 mAb is partially dependent upon expression of CD137 on NK cells, the magnitude and timing of CD137 expression in the peripheral blood or at the tumor site following antibody therapy may provide a predictive biomarker of response.

CD137 stimulation of NK cytotoxicity is restricted to HER2-overexpressing tumors by 2 mechanisms. First, CD137 upregulation requires contact with trastuzumab-coated tumor cells. For instance, HER2-negative tumor and/or irrelevant antitumor mAbs do not induce upregulation of CD137 on NK cells. Second, CD137 stimulation of activated (CD137 expressing) NK cells only enhances cytotoxicity toward mAb-coated tumor cells and not uncoated tumor cells. This explains why the enhanced functional activity we observed following in vitro and in vivo stimulation of activated NK cells with anti-CD137 agonistic mAbs maintained specificity to HER2-overexpressing tumors, including in an in vivo, 2-tumor model. This may translate to a clinical benefit in reduced toxicity of the combination antibody approach, as systemic NK cell stimulation should not be expected. Current therapies such as high-dose IL-2, though clinically beneficial, are limited by systemic toxicity $(36,37)$. Other approaches, such as IL-12 or blockade of inhibitory KIR receptors, similarly are unable to selectively target NK cells implicated in trastuzumab-mediated tumor cell killing $(36,38,39)$. In the approach developed here, we expect anti-CD137 mAbs to 
preferentially target activated NK cells (i.e., expressing CD137), including NK cells implicated in tumor-directed ADCC while sparing inactive or resting NK cells, thereby limiting potential offtarget toxicity of anti-CD137 mAbs.

In addition to dependence on trastuzumab and HER2-overexpressing breast cancer, the combination approach required proper sequence of antibody administration to demonstrate significant enhancement of trastuzumab's activity. In the absence of trastuzumab, we did not observe any level of anti-breast cancer activity of anti-CD137 mAbs either directly or indirectly by stimulation of NK cells in vitro or in vivo. If the murine model recapitulates the human clinical setting, at least in part, then determining this interval between antibodies is critical. Though a 24-hour interval allowed for peak CD137 expression in vitro following NK cell exposure to trastuzumab, based on the first 11 patients assessed prior to and following trastuzumab, the kinetics of CD137 expression are heterogeneous. Accrual of additional patients in the study of CD137 levels may identify the predictive factors. However, if current demographic and clinical-pathologic characteristics do not fully explain the variance, then administration of anti-CD137 mAbs could be individually tailored to the time point of maximal CD137 expression following trastuzumab.

To limit the potential effector cells responsible for breast cancer cell lysis, we used purified NK cells in vitro and, in vivo, partially immunodeficient mouse models. The $n u / n u$ and SCID mice are both competent in NK cells and macrophages and complement but lack a functional adaptive immune response. However, mAbs targeting CD137 in vivo likely influence the function of multiple cell types, including hematopoietic cells such as granulocytes, $\mathrm{T}$ cells, B cells, dendritic cells, and monocytes, in addition to NK cells, as well as nonhematopoietic cells $(25,40-43)$. In particular, our partially immunodeficient mouse models do not explore the impact of trastuzumab and anti-CD137 combination therapy on the adaptive immune response. This may be of importance, as we previously observed in an immunocompetent mouse model of lymphoma that anti-CD137 mAbs resulted in the generation of immune memory, as illustrated by protection from rechallenge (32). Recently, Bellati et al. detailed the importance of the adaptive immune response to trastuzumab therapy in its clinical efficacy (44). Taken together, we hypothesize that anti-CD137 agonistic $\mathrm{mAbs}$ following trastuzumab may also improve adaptive immunity in addition to enhancing ADCC. This hypothesis is supported by recent work by Stagg et al. (45). Recognizing that anti-CD137 $\mathrm{mAbs}$ enhance both adaptive and innate immune responses, $\mathrm{Fc}$ engineering may further improve efficacy by optimizing the binding to FcRIIB, recently recognized to be necessary for anti-TNFR mAbs' agonistic effects on adaptive immunity (46). Stimulation of CD137-expressing non-NK cells may also induce toxicity, including off-target hepatocyte toxicity due to CD8 T cell-dependent hepatitis, which has been observed in both mouse models and a phase I clinical trial of anti-CD137 monotherapy $(47,48)$. However, given the synergy between trastuzumab and anti-CD137 $\mathrm{mAbs}$, combination with a tumor-directed $\mathrm{mAb}$ may allow the use of minimal doses of anti-CD137 mAbs, limiting toxicities while retaining antitumor activity.

An area worthy of further investigation is the setting of CD137 stimulation in trastuzumab resistance. Clinical studies have been unable to demonstrate decreased HER2 receptor levels or blocked trastuzumab-binding capacity among patients with trastuzumabresistant disease $(4,49)$. Using a cell line with intrinsic resistance to trastuzumab, without loss of surface HER2 expression and due to either expression of t-DARPP (50) or E-cadherin (51), we observed preserved ability of anti-CD137 mAbs to enhance trastuzumabdependent cytotoxicity. Therefore, even in instances of diminished response to trastuzumab, enhancing ADCC with CD137 stimulation may offer the opportunity to overcome tumor resistance.

In conclusion, the results of our study demonstrate that antiCD137 mAbs can enhance trastuzumab's activity by stimulating the immune response to trastuzumab-bound, HER2-overexpressing breast cancer in vitro and in xenotransplant models. This approach is dependent upon sequential targeting first of the tumor by trastuzumab and second of the activated NK cells by anti-CD137 agonistic mAbs. This antibody strategy targeting both the tumor and the immune system may thus offer new opportunities to enhance the efficacy of therapeutic antibodies in cancer. This approach is widely applicable across cancers, including to known antibodies and known antigen-expressing tumors, such as rituximab and CD20-expressing lymphomas (32) and cetuximab and EGFR-expressing head and neck as well as colorectal tumors and to yet undeveloped antibodies to currently unknown tumor antigens. Clinical investigations are now warranted to determine whether this hypothesis holds true in patients.

\section{Methods}

Cell lines and culture. The human breast cancer cell lines BT474M1 and HER18 were obtained from Byron Hann at UCSF (San Francisco, California, USA). MCF7 and SKBR3 were obtained from James Ford at Stanford University. HCC1569, a HER2-expressing, trastuzumab-resistant breast cancer cell line, was obtained from Byran Hann at UCSF. The human CD20-positive B cell line, Raji, was obtained from ATCC. The SKBR3 cell line was cultured in McCoy's 5A modified medium (Life Technologies; Invitrogen) supplemented with $10 \%$ heat-inactivated FCS (HyClone Laboratories), $100 \mathrm{U} / \mathrm{ml}$ penicillin, and $100 \mu \mathrm{g} / \mathrm{ml}$ streptomycin (both from Life Technologies; Invitrogen). The MCF7 and BT474M1 cell lines were cultured in DMEM medium (Life Technologies: Invitrogen), Raji cell line was cultured in RPMI (Life Technologies; Invitrogen), and the HCC1569 and HER18 cell lines were cultured in DMEM/F12 1:1 medium (Life Technologies; Invitrogen), all supplemented as above. Cells were grown as adherent cultures at $37^{\circ} \mathrm{C}$ in $5 \% \mathrm{CO}_{2}$ and passaged after detachment by $0.05 \%$ trypsin (Life Technologies; Invitrogen). MCF7, BT474M1, HER18, and SKBR3 express minimal, moderate, and high levels of HER2, respectively, with specific fluorescence indices (tumor MFI/isotype MFI) of 0.17, 1.24, 1.54, and 1.62. These cell lines express neither the CD137 ligand nor the CD137 receptor.

Mice. Five- to six-week-old female athymic $(n u / n u)$ nude Foxn $1^{n u}$ and SCID mice (Prkdcsid) were purchased from Harlan and Jackson Laboratories and were housed at the Laboratory Animal Facility at the Stanford University Medical Center.

Therapeutic antibodies. Rat anti-mouse CD137 (4-1BB) mAb (IgG2a, clone 2A; ref. 52) was produced from ascites in SCID mice as previously described $(25,26)$. Control rat IgG was obtained from Sigma-Aldrich. Human antihuman CD137 mAb (BMS-663513, IgG4) was provided by Bristol-Myers Squibb. Rituximab, (murine-human chimeric anti-CD20, IgG1), trastuzumab (humanized anti-human epidermal growth factor receptor-2, IgG1), and trastuzumab D265A (variant of trastuzumab with a single alanine substitution that impairs binding of trastuzumab in all human FcrR; ref. 3) were obtained from Genentech.

CD137 expression on human NK cells from patient samples and healthy individuals. PBMCs, which were obtained from the Stanford Blood Center, were isolated from patients with HER $2 / \mathrm{neu}^{+}$breast cancer at 2 or 3 time points and from healthy donors by density gradient separation using Ficoll-Paque 
PLUS (Amersham Biosciences). PBMCs from patients with HER2/neu ${ }^{+}$ breast cancer at time points, including immediately before and either 4, 24, or 168 hours after trastuzumab infusion, were assayed for NK cell expression of CD137 and CD16. PBMCs isolated from healthy individuals or purified NK cells isolated by negative magnetic cell sorting using NK cell isolation beads (Miltenyi Biotec) were cultured, 1:1, in complete medium for 24 hours with breast cancer cell lines in the presence of rituximab, trastuzumab $(10 \mu \mathrm{g} / \mathrm{ml})$, or trastuzumab-D265A $(10 \mu \mathrm{g} / \mathrm{ml})$. Assessment of CD137 and CD16 expression on NK cells was performed in triplicate for each of 3 healthy individuals.

In vitro NK functional assays. PBMCs or purified NK cells were incubated for 24 hours with irradiated (50 Gy) HER2-expressing breast cancer cells (SKBR3 or HER18 or HCC1569) at a ratio of 1:1 and with trastuzumab $(10 \mu \mathrm{g} / \mathrm{ml})$. Following 24-hour culture of PBMCs with irradiated breast cancer cells, purified NK cells were then isolated by negative magnetic cell sorting using NK cell isolation beads (Miltenyi Biotec) according to the manufacturer's instructions and to greater than $90 \%$ purity, as defined by $\mathrm{CD}^{-}{ }^{-} \mathrm{CD} 56^{+}$and confirmed by flow cytometry. Activation of NK cells was confirmed by flow cytometry ( $>50 \%$ expression of CD137). The activated NK cells were then cultured with additional breast cancer cell lines (MCF7, BT474M1, HER18, SKBR3, or HCC1569) at a ratio of 1:1. After 18 hours, the supernatants were removed and assayed for IFN- $\gamma$ by ELISA (human IFN- $\gamma$ BD OptEIA ELISA Set and BD OptEIA Reagent Set B; BD Biosciences - Pharmingen). Cells from these cultures were incubated with FITC-annexin $\mathrm{V}$ and 7-AAD (both BD Biosciences) and analyzed by flow cytometry. Forward and side scatter were used to gate out the NK cells, and fluorescence was used to determine the percentage of apoptotic tumor cells by annexin $\mathrm{V}$ and 7-AAD staining. NK cell cytotoxicity was additionally measured by a chromium release assay (53): target tumor cells were labeled with $150 \mu \mathrm{Ci}{ }^{51} \mathrm{Cr}$ per $1 \times 10^{6}$ cells for 2 hours. Both activated purified and nonpurified NK cells were prepared as described above and were then added at variable effector/target cell ratios from 1:1 to 50:1. Percentage of lysis was determined after 4 hours of culture in the presence of anti-CD137 mAbs (BMS-663513, $10 \mu \mathrm{g} / \mathrm{ml}$ ) alone, trastuzumab $(10 \mu \mathrm{g} / \mathrm{ml})$ alone, or trastuzumab plus anti-CD137 mAbs (both at $10 \mu \mathrm{g} / \mathrm{ml}$ ). All assays were performed in triplicate with 3 independent NK cell samples.

Tumor transplantation and antibody therapy. BT474M1 breast cancer cells were implanted s.c. into 5- to 6-week-old female athymic $n u / n u$ mice at a dose of $5 \times 10^{6}$ cells in $50 \mu \mathrm{l}$ of PBS mixed with $50 \mu \mathrm{l}$ of Matrigel (BD Biosciences) 1 day after s.c. implantation of a $0.72 \mathrm{mg} / 60 \mathrm{~d}$ release $\beta$-estradiol pellet (Innovative Research of America). mAbs were administered by i.p. injections. Trastuzumab was given i.p. at $120 \mu \mathrm{g} /$ injection, and anti-CD137 $\mathrm{mAb}$ was given i.p. at $150 \mu \mathrm{g} /$ injection at various times after tumor inoculation. To determine the HER2 specificity of the therapy, $n u / n u$ mice were inoculated with $5 \times 10^{6}$ HER18 (HER2 overexpressing) breast tumor cells, s.c., on the right flank, and $5 \times 10^{6} \mathrm{MCF} 7$ (HER2 not overexpressing) breast tumor cells, s.c., on the left flank. Trastuzumab and anti-CD137 mAbs were administered after tumor inoculation as above. Tumor growth was measured by caliper twice a week and expressed as the product of length by width in square centimeters. Mice were sacrificed when tumor size reached $4 \mathrm{~cm}^{2}$ or when tumor sites ulcerated. All in vivo models were piloted with 5 mice per group and repeated with 10 mice per group (Figure 4, A-D, and Figure 5, A-C).

Primary breast cancer xenotransplant model and antibody therapy. Primary breast tumor tissue was obtained with informed consent from patients in accordance with the Declaration of Helsinki and study approval by Stanford University's Administrative Panels on Human Subjects. SU-258 was obtained from a primary tumor diagnosed as HER 2 overexpressing by FISH and negative for both estrogen and progesterone receptors. HER2 overexpression was confirmed by flow cytometry with specific fluorescence index (tumor MFI/isotype MFI) of 1.3 . SU-258 cells, $1 \times 10^{6}$, injected into 5- to 6 -week-old SCID mice by the intramammary route 24 hours after sublethal irradiation (200 cGy). On day 40, when tumor size averaged $0.25 \mathrm{~cm}^{2}$, mice were randomized to groups of 5 , each treated with trastuzumab followed the next day by anti-CD137 mAbs, or to control groups with single antibody treatments or no treatment. Treatment was repeated weekly in each group for a total of 3 treatments. Mice were monitored for tumor growth and survival as above. In vivo primary breast models were performed once with 5 mice per group (Figure 5D).

Antibodies for flow cytometry. $\mathrm{mAbs}$ to human antigens were used for staining of human PBMCs including CD16 FITC, CD137 ligand PE, CD56 PE, CD3 PerCP, anti-HER2/neu APC, CD137 APC, APC-H7, FITC-annexin $\mathrm{V}$, and 7-AAD (all from BD Biosciences). Stained cells were collected on a FACSCalibur or a LSRII 3-laser cytometer (BD), and data were analyzed using Cytobank (http://www.cytobank.org) (54).

Statistics. Prism software (GraphPad) was used to analyze tumor growth and determine statistical significance of differences (including mean \pm SEM) between groups by applying a 2-tailed, unpaired Student's $t$ test or 2-way ANOVA with Bonferroni's correction for multiple comparisons. KaplanMeier plots were used to analyze survival. Comparisons of survival curves were made using the log-rank test. $P<0.05$ was considered significant. For tumor burdens, comparisons of means were done by ANOVA.

Study approval. Human peripheral blood and primary breast tumor samples were obtained following informed consent from patients in accordance with the Declaration of Helsinki by Stanford University's Administrative Panels on Human Subjects (NCT01114256). All animal experiments were approved by the Stanford Administrative Panel on Laboratory Animal Care and conducted in accordance with NIH guidelines.

\section{Acknowledgments}

This work was supported by the California Breast Cancer Research Program, EUREKA (R01 CA153248-01), the Reliable Cancer Therapy Fund, and Nadia's Gift Foundation at the Stanford Cancer Center. M.J. Goldstein is supported by the Howard Hughes Medical Institute Research Training Fellowship. R. Houot is supported by fellowships from the Foundation de France and the Association pour la Recherche sur le Cancer. H.E. Kohrt is supported by fellowships from the American Society of Hematology and the American Society of Clinical Oncology and a Department of Defense Breast Cancer postdoctoral fellowship award (no. 26687). K. Weiskopf is supported by the Stanford University Medical Scientist Training Program. R. Levy is an American Cancer Society Clinical Research Professor.

Received for publication September 30, 2011, and accepted in revised form January 4, 2012.

Address correspondence to: Ronald Levy, Division of Oncology, 269 Campus Drive, CCSR 1105, Stanford University Medical Center, Stanford, California 94305-5151, USA. Phone: 650.725.6452; Fax: 650.736.1454; E-mail: levy@stanford.edu.
1. Hall PS, Cameron DA. Current perspective - trastuzumab. Eur J Cancer. 2009;45(1):12-18.

2. Varchetta $S$, et al. Elements related to heterogeneity of antibody-dependent cell cytotoxicity in patients under trastuzumab therapy for primary oper- able breast cancer overexpressing Her2. Cancer Res. 2007;67(24):11991-11999.

3. Clynes RA, Towers TL, Presta LG, Ravetch JV. Inhibitory $\mathrm{Fc}$ receptors modulate in vivo cytoxicity against tumor targets. Nat Med. 2000;6(4):443-446.
4. Gennari R, et al. Pilot study of the mechanism of action of preoperative trastuzumab in patients with primary operable breast tumors overexpressing HER2. Clin Cancer Res. 2004;10(17):5650-5655.

5. Menard S, et al. Apoptosis induction by trastuzumab: 
possible role of the core biopsy intervention. J Clin Oncol. 2005;23(28):7238-7240.

6. Caligiuri MA. Human natural killer cells. Blood. 2008;112(3):461-469.

7. Tamura K, et al. Fc $\{$ gamma\}R2A and $3 \mathrm{~A}$ polymorphisms predict clinical outcome of trastuzumab in both neoadjuvant and metastatic settings in patients with HER2-positive breast cancer. Ann Oncol. 2011;22(6):1302-1307.

8. Musolino A, et al. Immunoglobulin G fragment $\mathrm{C}$ receptor polymorphisms and clinical efficacy of trastuzumab-based therapy in patients with HER-2/ neu-positive metastatic breast cancer. J Clin Oncol. 2008;26(11):1789-1796.

9. Gluck WL, et al. Phase I studies of interleukin (IL)-2 and rituximab in B-cell non-Hodgkin's lymphoma: IL-2 mediated natural killer cell expansion correlations with clinical response. Clin Cancer Res. 2004. 10(7):2253-2264

10. Ansell SM, et al. Phase 1 study of interleukin-12 in combination with rituximab in patients with B-cell non-Hodgkin lymphoma. Blood. 2002;99(1):67-74.

11. Lin W, et al. Fc-dependent expression of CD137 on human NK cells: insights into "agonistic" effects of anti-CD137 monoclonal antibodies. Blood. 2008; 112(3):699-707.

12. Benz CC, et al. Estrogen-dependent, tamoxifenresistant tumorigenic growth of MCF-7 cells transfected with HER2/neu. Breast Cancer Res Treat. 1992; 24(2):85-95

13. Smith I, et al. 2-year follow-up of trastuzumab after adjuvant chemotherapy in HER2-positive breast cancer: a randomised controlled trial. Lancet. 2007; 369(9555):29-36

14. Bedard P, de Azambuja E, Cardoso F. Beyond trastuzumab: overcoming resistance to targeted HER-2 therapy in breast cancer. Curr Cancer Drug Targets. 2009;9(2):148-162.

15. Spector NL, Blackwell KL. Understanding the mechanisms behind trastuzumab therapy for human epidermal growth factor receptor 2-positive breast cancer. J Clin Oncol. 2009;27(34):5838-5847.

16. Kiewe P, et al. Phase I trial of the trifunctional anti-HER $2 x$ anti-CD3 antibody ertumaxomab in metastatic breast cancer. Clin Cancer Res. 2006 ; 12(10):3085-3091.

17. Beeram M, et al. A phase I study of trastuzumabMCC-DM1 (T-DM1), a first-in-class HER2 antibodydrug conjugate (ADC), in patients (pts) with HER2+ metastatic breast cancer (BC). J Clin Oncol. 2007; 25(18 suppl):abstr 1042 .

18. Baselga J, et al. Phase II trial of Pertuzumab and Trastuzumab in patients with human epidermal growth factor receptor positive metastatic breast cancer that progressed during prior Trastuzumab therapy. J Clin Oncol. 2010;28(7):1138-1144.

19. Newton RC, et al. Clinical benefit of INCB7839, a potent and selective ADAM inhibitor, in combination with trastuzumab in patients with metastatic HER2+ breast cancer. J Clin Oncol. 2010; 28(15 suppl):abstr 3025 .

20. Salazar LG, et al. Persistent immunity and survival afterimmunization with a HER2/neu(HER2) vaccine. J Clin Oncol. 2009;27(15suppl):abstr 3057.

21. Melero I, Hervas-Stubbs S, Glennie M, Pardoll DM, Chen L. Immunostimulatory monoclonal antibodies for cancer therapy. Nat Rev Cancer. 2007;7(2):95-106.

22. Takeda $\mathrm{K}$, et al. Combination therapy of established tumors by antibodies targeting immune activating and suppressing molecules. J Immunol. 2010;184(10):5493-5501.

23. Murillo O, et al. Therapeutic antitumor efficacy of anti-CD137 agonistic monoclonal antibody in mouse models of myeloma. Clin Cancer Res. 2008; 14(21):6895-6906.

24. Wilcox RA, et al. Cutting edge: Expression of functional CD137 receptor by dendritic cells. JImmunol. 2002;168(9):4262-4267.

25. Narazaki H, Zhu Y, Luo L, Zhu G, Chen L. CD137 agonist antibody prevents cancer recurrence: contribution of CD137 on both hematopoietic and nonhematopoietic cells. Blood. 2010;115(10):1941-1948.

26. Houot R, et al. Therapeutic effect of CD137 immunomodulation in lymphoma and its enhancement by Treg depletion. Blood. 2009;114(16):3431-3438.

27. Baessler T, et al. CD137 ligand mediates opposite effects in human and mouse NK cells and impairs NK cell reactivity against human acute myeloid leukemia cells. Blood. 2010;115(15):3058-3069.

28. Melero I, Johnston JV, Shufford WW, Mittler RS, Chen L. NK1.1 cells express 4-1BB (CDw137) costimulatory molecule and are required for tumor immunity elicited by anti-4-1BB monoclonal antibodies. Cell Immunol. 1998;190(2):167-172.

29. Wilcox RA, Tamada K, Strome SE, Chen L. Signaling through NK cell-associated CD137 promotes both helper function for CD8+ cytolytic T cells and responsiveness to IL-2 but not cytolytic activity. J Immunol. 2002;169(8):4230-4236

30. Maniar A, et al. Human \{gamma\}\{delta\} T lymphocytes induce robust NK cell mediated antitumor cytotoxicity through CD137 engagement. Blood. 2010;116(10):1726-1733

31. Gong W, et al. Immobilized MHC class I chainrelated protein A synergizes with $\mathrm{IL}-15$ and soluble 4-1BB ligand to expand NK cells with high cytotoxicity ex vivo. Cell Mol Immunol. 2010; $7(6): 477-484$

32. Kohrt HE, et al. CD137 stimulation enhances the anti-lymphoma activity of anti-CD20 antibodies. Blood. 2011;117(8):2423-2432.

33. Cooper MA, Fehniger TA, Caligiuri MA. The biology of human natural killer-cell subsets. Trends Immunol. 2001;22(11):633-640.

34. Bowles JA, Weiner GJ. CD16 polymorphisms and NK activation induced by monoclonal antibody-coated target cells. J Immunol Methods. 2005; 304(1-2):88-99.

35. Juelke $\mathrm{K}$, et al. CD62L expression identifies a unique subset of polyfunctional CD56dim NK cells. Blood. 2010;116(8):1299-1307.

36. Mani A, et al. A phase II trial of trastuzumab in combination with low-dose interleukin-2 (IL-2) in patients (PTS) with metastatic breast cancer (MBC) who have previously failed trastuzumab. Breast Cancer Res Treat. 2009;117(1):83-89.

37. Acquavella $\mathrm{N}$, et al. Toxicity and activity of a twice daily high-dose bolus interleukin 2 regimen in patients with metastatic melanoma and metastatic renal cell cancer. J Immunother. 2008;31(6):569-576.

38. Bekaii-Saab TS, et al. A phase I trial of paclitaxel and trastuzumab in combination with interleukin-12 in patients with HER2/neu-expressing malignancies. Mol Cancer Ther. 2009;8(11):2983-2991.

39. Srivastava S, Feng H, Zhang S, Liang J, Squiban P, Farag S. Enhancing natural killer (NK) cell mediated killing of non-Hodgkin's lymphoma. ASH Annual Meeting Abstracts. 2009;114:2706.

40. Jiang D, Schwarz H. Regulation of granulocyte and macrophage populations of murine bone marrow cells by G-CSF and CD137 protein. PLoS One. 2010; 5(12):e15565

41. Zhang X, et al. CD137 promotes proliferation and survival of human B cells. J Immunol. 2010; 184(2):787-795

42. Murillo O, et al. In vivo depletion of DC impairs the anti-tumor effect of agonistic anti-CD137 mAb. Eur J Immunol. 2009;39(9):2424-2436.

43. Sharma RK, et al. 4-1BB ligand as an effective multifunctional immunomodulator and antigen delivery vehicle for the development of therapeutic cancer vaccines. Cancer Res. 2010;70(10):3945-3954.

44. Bellati F, Napoletano C, Ruscito I, Liberati M, Panici PB, Nuti M. Cellular adaptive immune system plays a crucial role in trastuzumab clinical efficacy. J Clin Oncol. 2010;28(21):e369-e370.

45. Stagg J, et al. Anti-ErbB-2 mAb therapy requires type I and II interferons and synergizes with antiPD-1 or anti-CD137 mAb therapy. Proc Natl Acad Sci US A. 2011;108(17):7142-7147.

46. Li F, Ravetch JV. Inhibitory Fcgamma receptor engagement drives adjuvant and anti-tumor activities of agonistic CD40 antibodies. Science. 2011;333(6045):1030-1034.

47. Dubrot J, et al. Treatment with anti-CD137 mAbs causes intense accumulations of liver $\mathrm{T}$ cells without selective antitumor immunotherapeutic effects in this organ. Cancer Immunol Immunother. 2010; 59(8):1223-1233.

48. Sznol M, et al. Phase I study of BMS-663513, a fully human anti-CD137 agonist monoclonal antibody, in patients (pts) with advanced cancer (CA). J Clin Oncol. 2008;26(20 suppl):abstr 3007.

49. Mohsin SK, et al. Neoadjuvant trastuzumab induces apoptosis in primary breast cancers. JClin Oncol.2005; 23(11):2460-2468.

50. Belkhiri A, et al. Expression of t-DARPP mediates trastuzumab resistance in breast cancer cells. Clin Cancer Res. 2008;14(14):4564-4571.

51. Yamauchi $\mathrm{C}$, et al. E-cadherin expression on human carcinoma cell affects trastuzumab-mediated antibody-dependent cellular cytotoxicity through killer cell lectin-like receptor G1 on natural killer cells. Int J Cancer. 2011;128(9):2125-2137.

52. Wilcox RA, et al. Provision of antigen and CD137 signaling breaks immunological ignorance, promoting regression of poorly immunogenic tumors. J Clin Invest. 2002;109(5):651-659.

53. Kim GG, Donnenberg VS, Donnenberg AD, Gooding W, Whiteside TL. A novel multiparametric flow cytometry-based cytotoxicity assay simultaneously immunophenotypes effector cells: comparisons to a 4 h 51Cr-release assay. J Immunol Methods. 2007; 325(1-2):51-66

54. Kotecha N, et al. Single-cell profiling identifies aberrant STAT5 activation in myeloid malignancies with specific clinical and biologic correlates. Cancer Cell. 2008;14(4):335-343. 